\title{
Non-Affine Nonlinear Systems Adaptive Optimal Trajectory Tracking Controller Design and Application
}

\author{
Haoping WANG ${ }^{1, *}$, Yang TIAN ${ }^{1}$, Christian VASSEUR ${ }^{2}$ \\ 1 Sino-French International Joint Laboratory of Automatic Control and Signal Processing (LaFCAS), \\ Nanjing University of Science \& Technology (NUST), \\ Nanjing 210094, China \\ ${ }^{2}$ LAGIS- CNRS UMR 8219, LaFCAS, \\ University Lille Nord de France, \\ Lille France, 59600
}

Corresponding author: Haoping WANG, hp.wang@ujust.edu.cn

\begin{abstract}
For considering a general non-affine in control nonlinear systems, this present paper introduces a new Adaptive Nonlinear Optimal Recursive Control (ANORC) used for trajectory tracking. This referred ANORC controller which is developed on a $L_{2}$ norm prescribed to minimize a trajectory tracking error has advantages of adaptive turning and optimal features. Furthermore, under the feedback of the systems output, this ANORC which adopts a control structure in recursive scheme and employs no knowledge of internal dynamics ensures the realization of the systems output trajectory tracking. Then to demonstrate the performances and effectiveness of this proposed ANORC controller, three different academic numerical examples (in the form of Single Input Single Output (SISO), Multi-Input Single Output (MISO) and Multi-Input Multi-Output (MIMO)) are implemented.
\end{abstract}

Keywords: Adaptive nonlinear optimal control, non-affine in control nonlinear systems, model free recursive control; trajectory tracking.

\section{Introduction}

During the last decades, the control of nonlinear systems which concerns normally the stabilization or trajectory tracking control is generally considered difficult and becomes worldly active and hot research subjects [1-5]. The combined consideration of twofold for a controlled systems, which means the realization not only the control (trajectory tracking or stabilization), but also the optimization of a prescribed performance index (such as minimization the trajectory tracking error, the fuel consumption, or particles emissions, etc) are not easy. Currently there exists no adequate work relating the optimal control of nonlinear systems, especially for the non-affine in control nonlinear systems, since their corresponding Hamilton-Jacobi- Bellman (HJB) equation are difficult to resolve [6-7].

Compared to linear or nonlinear affine in control systems to solve their corresponding Riccati Equation (RE), Algebraic Riccati Equation (ARE) and HJB, the nonlinear nonaffine in control systems are more complex and has no feasible solutions when their systems dynamics are not explicit. Recently, online adaptive approximation- based optimal control which refers to an online approximator based Adaptive Critic Designs (ACD) is gradually proposed [8-9]. While in reference
[5], a relative new optimal adaptive control (OAC) is proposed for MIMO nonlinear systems in the form of strict feedback. The optimal adaptive feedback scheme is introduced for the affine systems to estimate the solution of HJB equation online which becomes the optimal feedback control input for the closed-loop system.

In adaptive control literature, various nonlinear systems and methods are appeared and discussed [10]. Nonlinear systems in form of strict feedback in a variety of ways and their stability are studied with the application of the normal Backstepping scheme without any optimality. One of the most popular approaches is called "dynamic inversion and feedback linearization". Recently, the inverse optimal control for strict feedback systems which consist on an associated cost function based control law is introduced in [11]. Furthermore, in many applications, the inverse function for nonlinear systems is difficult or even impossible to find. In many practical cases, systems full states are not fully accessible. Then output feedback nonlinear controllers which use model-based observers were developed when their systems dynamics are known. While in many cases where their systems dynamics are unknown, the control of unknown strict feedback systems using adaptive neural-network based scheme is given to approximate their uncertain dynamics [5]. 
Therefore in our previous paper, a composed adaptive controller which is based on a recursive model free stabilization subcontroller (RMFSSC) and a recursive uncertain dynamic compensation subcontroller (RUDCSC) are proposed for nonlinear systems in [12]. The RMFSSC is a recursive model free controller based on the theory of Piecewise-Continuous Systems (PCS) which are a particular class of hybrid systems with autonomous switching and controlled impulses [13-17]. Using PCS theory, piecewise-continuous controllers were firstly developed to enable sampled trajectory tracking of linear systems [13-17]. Then for improving trajectory tracking performance, a derived piecewise continuous controller and recursive model free controller were proposed in [15-16]. Unfortunately, the referred proposed RMFSSC is defined without any prescribed cost function to minimize and not considered for the case of non-affine in control nonlinear systems. Thus in this present paper for non-affine in control nonlinear systems, a new Adaptive Nonlinear Optimal trajectory tracking Recursive Controller (ANORC) is proposed. This referred ANORC controller which employs only the output of the non-affine in control nonlinear systems and a $L_{2}$ norm to minimize the trajectory tracking error has a relative simple architecture and can be implemented to the case where the internal dynamics and parameters of the controlled non-affine in control nonlinear systems are unknown.

The following paper is organized as: in section 2 , the research problem of the considered trajectory tracking of non-affine in control nonlinear systems is introduced. Then in Section 3, a two steps design approach of adaptive nonlinear optimal trajectory tracking recursive controller is developed. After that in Section 4 based on the proposed method, three academic numerical examples are implemented to validate the proposed method performance and robustness. Finally it is followed by some conclusion remarks in Section 5.

\section{Problem Statement}

In this paper, the following general nonlinear non-affine in control system is considered as follows:

$$
\dot{x}(t)=f(x(t), u(t), t)
$$

where $\quad x(t) \in \Re^{n}, \quad u(t) \in \Re^{r} \quad$ and $f(x(t), u(t), t)$ are respectively the systems state, input control signal, and dynamics supposed to be derivative with respect to $u(t)$.

And the system corresponding output is assumed in the following form

$y(t)=C x(t)$

where $C \in \Re^{m \times n}$ represents the system output matrix and $y(t) \in \Re^{m}$ is the system output which is supposed to be measurable.

The control objective of this paper is to realize a feasible trajectory tracking controller which ensures the following defined trajectory tracking error trends to zero

$\lim _{t \rightarrow \infty}\left(e_{y}(t)=c_{y}(t)-y(t)\right) \rightarrow 0$

where $c_{y}(t)$ is the desired output reference and supposed to be differentiable. Noting that $c_{\dot{y}}(t)=\frac{d c_{y}(t)}{d t}$ is the derivative signal of desired output reference $c_{y}(t)$.

\section{Output Trajectory Tracking Controller Development}

In order to realize the above trajectory tracking controller, a two-step design approach is proposed as follows:

Firstly, a $L_{2}$ norm based Intermediate Optimal Controller (IOC) is designed to minimize the trajectory tracking error between the derivative desired output reference $c_{\dot{y}}(t)$ and output derivative $\dot{y}(t)$,

Secondly, based on the developed IOC controller, an Adaptive Optimal Recursive Controller (AORC) is developed which can be used to realize the refereed control objective in (3). Thus the entire proposed controller is called Adaptive Nonlinear Optimal trajectory tracking Controller (ANOC).

\subsection{First step: intermediate optimal controller design}

By recalling the defined non-affine in control nonlinear system (1), the following intermediate trajectory tracking error which concerns the system output derivative and its corresponding reference can be considered as

$e_{\dot{y}}(t)=c_{\dot{y}}(t)-C f(x, u, t)$ 
With the introduced notations of $z=c_{\dot{y}}$, one has the following $L_{2}$ norm based error

$$
\begin{aligned}
& \left\|e_{\dot{y}}^{2}\right\|=e_{\dot{y}}^{T}= \\
& (z-C f(x, u, t))^{T}(z-C f(x, u, t))
\end{aligned}
$$

which can be calculated in further as follow

$\left\|e_{\dot{y}}^{2}\right\|=z^{T} z-z^{T} C f(x, u, t) \quad-$

$f^{T}(x, u, t) C^{T} z+f^{T}(x, u, t) C^{T} C f(x, u, t)$.

By differentiating the above $L_{2}$ norm with respect to $u(t)$, one has the following relationship as

$0=-2 \nabla_{u} f^{T} C^{T} z+2 \nabla_{u} f^{T} C^{T} C f(x, u, t)$

with

$$
\nabla_{u} f=\left[\begin{array}{cccc}
\frac{\partial f_{1}}{\partial u_{1}} & \frac{\partial f_{1}}{\partial u_{2}} & \cdots & \frac{\partial f_{1}}{\partial u_{r}} \\
\frac{\partial f_{2}}{\partial u_{1}} & \frac{\partial f_{2}}{\partial u_{2}} & \cdots & \frac{\partial f_{2}}{\partial u_{r}} \\
\vdots & \vdots & \vdots & \vdots \\
\frac{\partial f_{n}}{\partial u_{1}} & \frac{\partial f_{n}}{\partial u_{2}} & \cdots & \frac{\partial f_{n}}{\partial u_{r}}
\end{array}\right]
$$

Then from the above equation (6), one can deduce

$\nabla_{u} f^{T} C^{T}(z-C f(x, u, t))=0$

which means with the assumption of $\nabla_{u} f^{T} C^{T} \neq 0$, one has the following obtained IOC controller

$c_{\dot{y}}-C f(x, u, t)=0$

It is important to remind that the above equation which is an implicit equation with respect to $u(t)$, can't be generally possible to give an explicit expression of $u(t)$. Thus without knowing how to calculate the control input $u(t)$, with the introduced equations of (1) and (8), one achieves the trajectory tracking of the systems desired output derivative reference $\dot{y}(t) \equiv c_{\dot{y}}(t)$.

\subsection{Second step: output recursive adaptive controller design}

\subsubsection{Trajectory tracking controller design}

To ensure the trajectory tracking of $c_{y}(t)$ to $y(t)$, the following relationship is to be proposed with an additional new term to equation (8)

$c_{\dot{y}}-C f(x, u, t)-\beta\left(c_{y}-y\right)=0$ where $\beta \in \Re^{m \times m}$ is a square matrix and selected with stable eigenvalues.

The above equation can be simplified with relationship of $\dot{y}(t)=C f(x, u, t)$ as follows

$\left(c_{\dot{y}(t)}-\dot{y}(t)\right)=\beta\left(c_{y}(t)-y(t)\right)$

As mentioned in the beginning of this subsection, because of the selected stable matrix $\beta$ with appropriate stable eigenvalues, the trajectory tracking of $\left(c_{y}(t) \rightarrow y(t)\right)$ can be ensured.

For simplification, the stable matrix can be selected in the form of $\beta=-k^{2} I_{m}$ where $k$ and $I_{m}$ are respectively a positive constant and a $m$-dimensional unit identity matrix.

\subsubsection{Implicit representation problem of $u(t)$}

One recalls that the defined control objective is to minimize the trajectory tracking error between the desired output reference and its corresponding output signal. For real time implementation, one needs to resolve the equation (9) and to find its corresponding explicit representation form, but unfortunately this resolution is not always exits. To remove this inconvenient, here a recursive calculation structure method is proposed to remove this incontinent.

With the introduced notations of $F(x, u, t)=C f(x, u, t) \quad$ and $\quad Z(t)=c_{\dot{y}}(t)$ $-\beta\left(c_{y}(t)-y(t)\right)$, equation (9) can be rewritten as $Z-F(x, u, t)=0$ which can be denoted further like

$$
u(t)=\lim _{\eta^{2} \rightarrow 0}\left(\left(1-\eta^{2}\right) u(t)+d(Z(t)-F(z, u, t))\right)
$$

where matrix $d$ is a full-rank matrix with dimensions of $r \times m$, and the $\eta$ is constant with small value.

Finally according to (11), the following modified output based recursive adaptive control can be proposed as follows

$$
\begin{aligned}
& u(t)=\lim _{\eta^{2} \rightarrow 0}\left(\left(1-\eta^{2}\right) u(t)\right. \\
& +d\left(\left(c_{\dot{y}}(t)-\dot{y}(t)\right)-d \beta\left(c_{y}(t)-y(t)\right)\right)
\end{aligned}
$$

It is important to note that under the high gain case which means $k^{2}$, the term of $\left(c_{\dot{y}}-\dot{y}\right)$ in (12) can be neglected with respect to $\beta\left(c_{y}-y\right)$ and by selection of $\beta=-k^{2} . I_{m}$, one obtains the ANORC controller

$$
\begin{aligned}
& u(t)=\lim _{\eta^{2} \rightarrow 0}\left(\left(1-\eta^{2}\right) u(t)+\right. \\
& k^{2} d\left(c_{y}(t)-y(t)\right)
\end{aligned}
$$




\subsubsection{Consideration of real-time implementation for ANORC controller}

The above ANORC controller which is illustrated in Figure 1, has two recursive calculation loops: the first concerns the realization of a high gain $k^{2}$ which is similar to the introduced derived PCC controller in [15], and the second relates to $u(t)$ whose realization needs the introduction of a second very small parameter which is denoted as $\eta^{2}$.

Reminding that with the implementation of this proposed ANORC controller, the knowledge of internal dynamics and parameters is not necessary.

\section{Illustrative Examples}

In this section, to illustrate the presented ANORC method, the following three examples have been tested.

\subsection{First example of a SISO system}

To illustrate the validity of the proposed method, a SISO nonlinear non-affine in control system which is defined as follows

$\dot{x}_{1}(t)=x_{2}(t)$

$\dot{x}_{2}(t)=x_{1}^{2}+0.15 u^{3}+0.1\left(1+x_{2}^{2}\right) u+$ $\sin (0.1 u)+d(t) y(t)=x_{1}(t)$

is considered, where $d(t)=0.5 \sin (10 t)$ is an external disturbance. And the proposed desired trajectory reference is defined as

$y_{d}=\sin (t)+\sin (0.5 t)$.
And their corresponding numerical results are illustrated in Figures 2 - 4 which represent respectively the output trajectory tracking, output derivative tracking, and the input signal. Noting the proposed ANORC controller ensures very well the trajectory tracking of desired references.

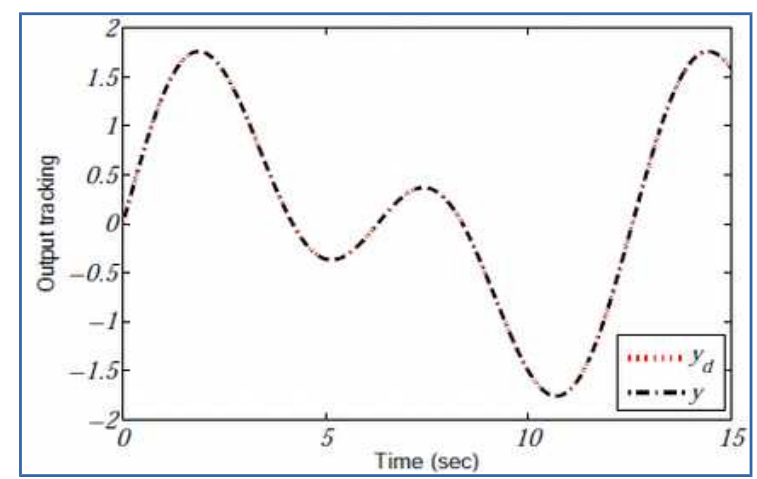

Figure 2. Output trajectory tracking

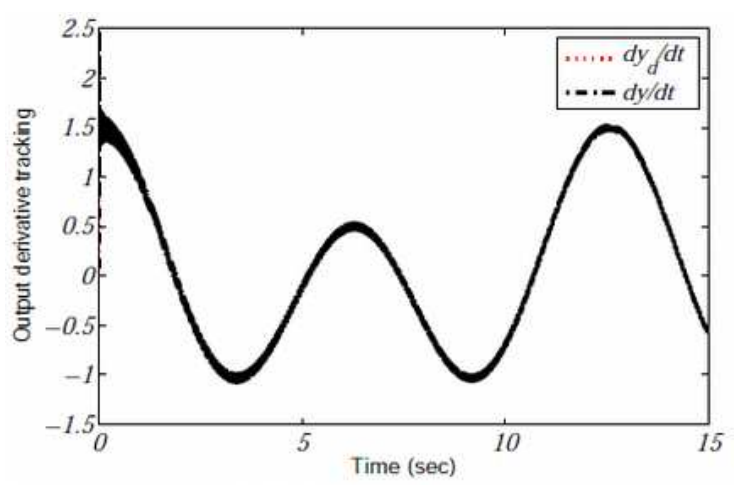

Figure 3. Output derivative tracking

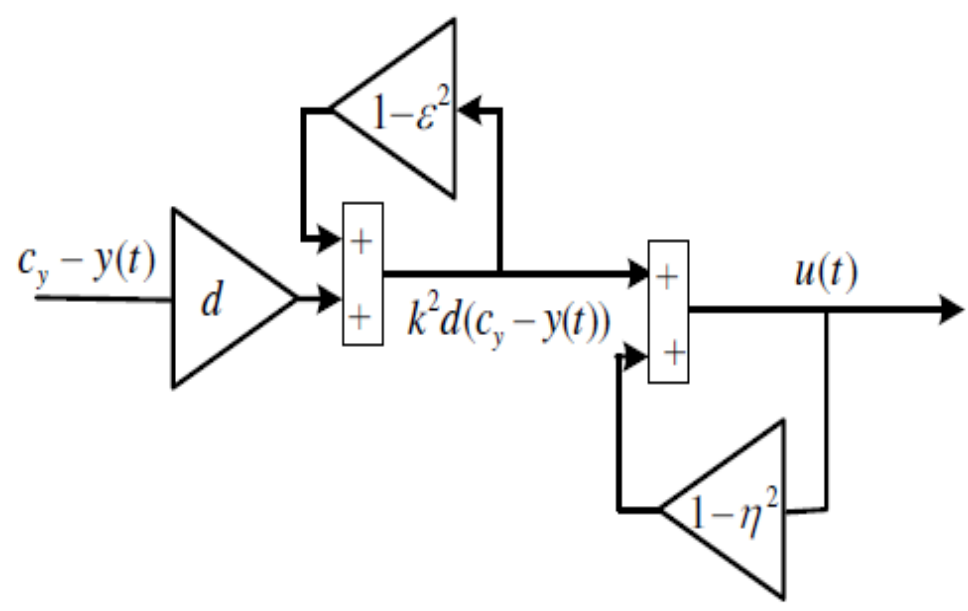

Figure 1. Double recursive High Gain 


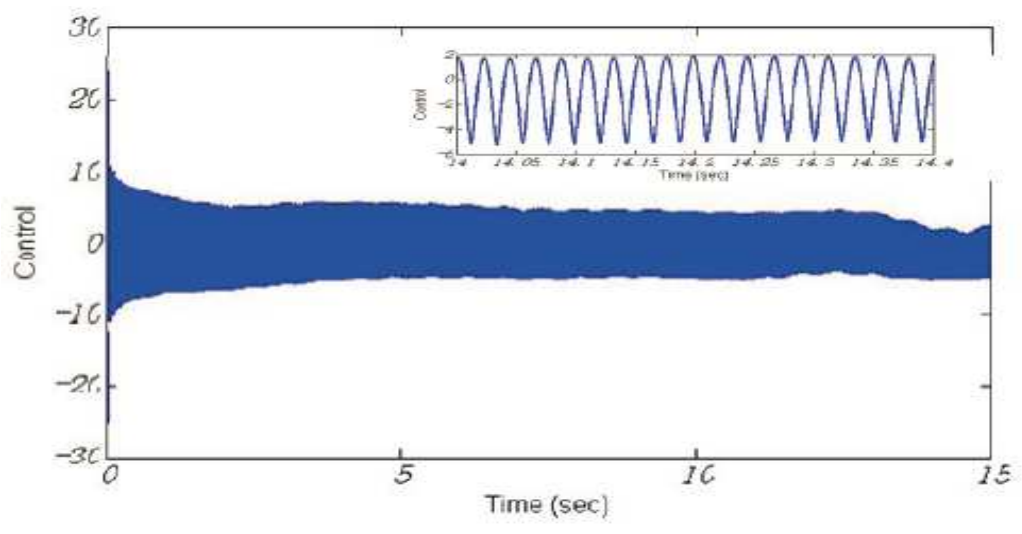

Figure 4. Input control $u_{1}$.

\subsection{Second example of a MISO system}

Then, the second considered example is an academic numerical system which is underactuated and unstable is considered [6]

$\dot{x}_{1}(t)=u_{1}(t)+2 x_{3}(t) u_{2}(t)$

$\dot{x}_{2}(t)=t x_{3}(t)+2\left(x_{1}(t)+1\right) u_{1}(t)+$

$4 x_{1}(t) x_{3}(t) u_{2}(t)$

$\dot{x}_{3}(t)=u_{2}(t)$

and the system output is defined as follows

$y(t)=\left[\begin{array}{lll}1 & 1 & -x_{3}\end{array}\right]\left[\begin{array}{lll}x_{1} & x_{2} & x_{3}\end{array}\right]^{T}=x_{1}(t)+$ $x_{2}(t)-x_{3}^{2}(t)$.

Let $x(t)=\left[\begin{array}{lll}x_{1}(t) & x_{2}(t) & x_{3}(t)\end{array}\right]^{T}, u(t)=\left[\begin{array}{ll}u_{1} & u_{2}\end{array}\right]$, and $C(x, t)=\left[\begin{array}{lll}1 & 1 & -x_{3}\end{array}\right]$. And the desired trajectory tracking reference is defined as a 3rdorder Bézier polynomial as follows

$$
\begin{aligned}
& c_{y}(t)=0.5\left((\sin (1.05 t))^{3}-\right. \\
& \left.3(\sin (1.05 t))^{2}+3 \sin (1.05 t)\right) .
\end{aligned}
$$

Reminding that the above numerical example is an under-actuated unstable nonlinear system which is not easy to control, and the parameters in the considering controller (13) are $\eta=0.2$, $d=\left[\begin{array}{ll}1 & 1\end{array}\right]^{T}, \quad$ and $\quad \beta=\left[\begin{array}{ll}2 & 1\end{array}\right]^{T} . \quad$ Their corresponding results are illustrated in Figure 5-7. From Figure 5 and Figure 6, it can be concluded clearly that the above intermediate optimal controller based output controller ensures not only the perfect trajectory tracking of the nonlinear non-affine in control systems output trajectory, but also the systems output derivative. And considering the control input signals which are illustrated in Figure 7, the proposed ANORC controller ensures the stability of the entire controlled nonlinear non-affine in control system whose state are bounded.

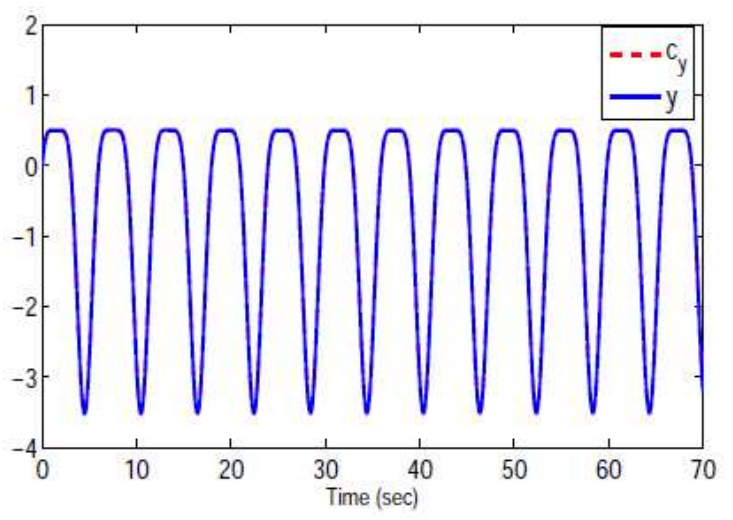

Figure 5. Output trajectory tracking

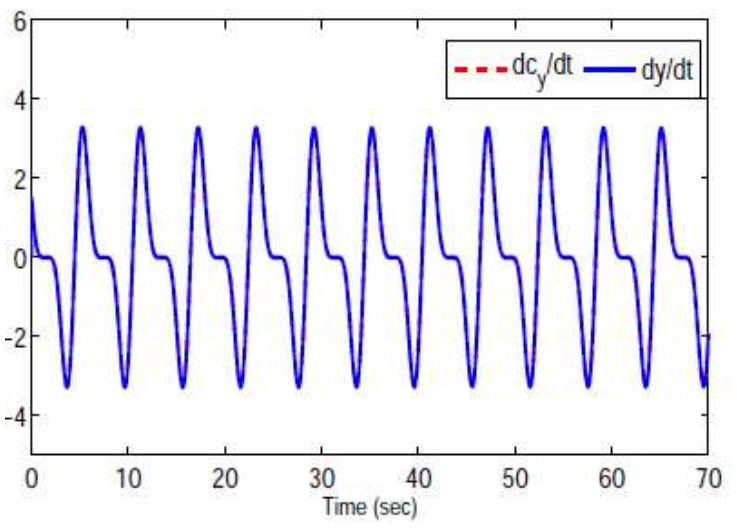

Figure 6. Output derivative tracking

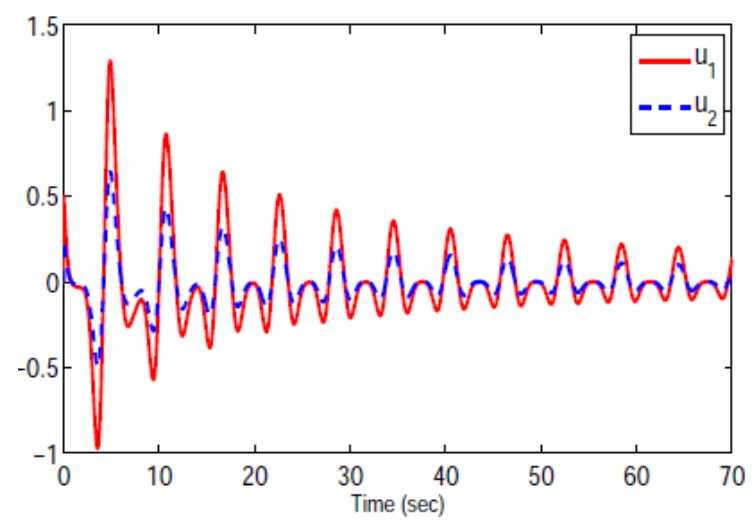

Figure 7. Input control signals $\left(u_{1}, u_{2}\right)$ 


\subsection{Third example of a MIMO system}

Finally, the third considered example is a MIMO system which is taken from [18] defined as follows

$$
\begin{aligned}
& \dot{x}_{1}(t)=u_{1}(t)+x_{2}(t) x_{3}(t) u_{2}^{4}(t) \\
& \dot{x}_{2}(t)=x_{1}(t) u_{2}(t)+x_{3}^{2}(t) u_{2}^{3}(t) \\
& \dot{x}_{3}(t)=u_{2}(t)
\end{aligned}
$$

The system output is defined as follows $y_{1}(t)=x_{1}, y_{2}(t)=x_{2}$.

And the desired trajectory tracking reference is defined as

$$
\begin{aligned}
& {\left[\begin{array}{ll}
y_{d 1} & y_{d 2}
\end{array}\right]^{T}=\left[\begin{array}{ll}
5+\sin (2 t+\pi / 2) & 2 \sin (2 t)+ \\
\sin (4 t)
\end{array}\right]^{T}}
\end{aligned}
$$

Under these conditions, their corresponding numerical results are illustrated in Figure 8Figure 11. With the above selected systems output references, the Figure 8 and Figure 9 represents respectively the output and output derivative trajectory tracking. And the entire state of the controlled non-affine in control nonlinear system is illustrated in Figure 10 which demonstrates well the stabilization of the entire controlled systems. And the proposed ANORC multi-input signals are shown in Figure 11.

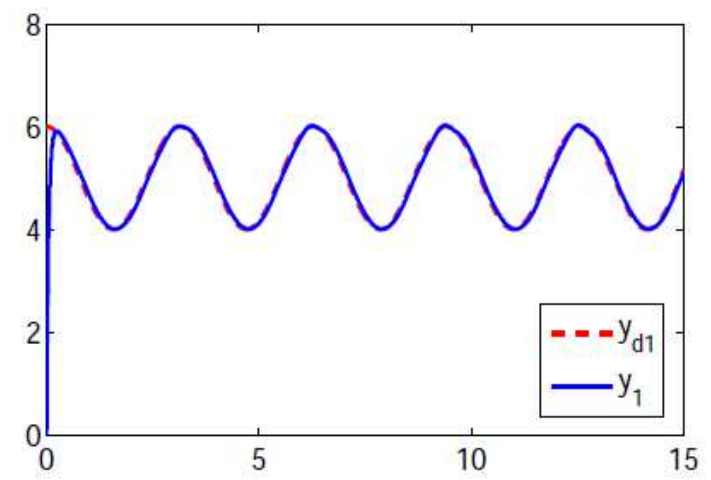

Figure 8. Output trajectory tracking

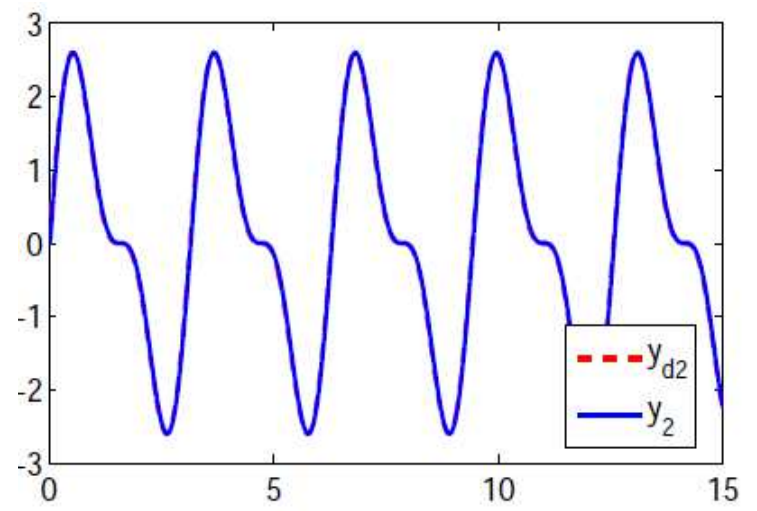

Figure 9. Output derivative tracking

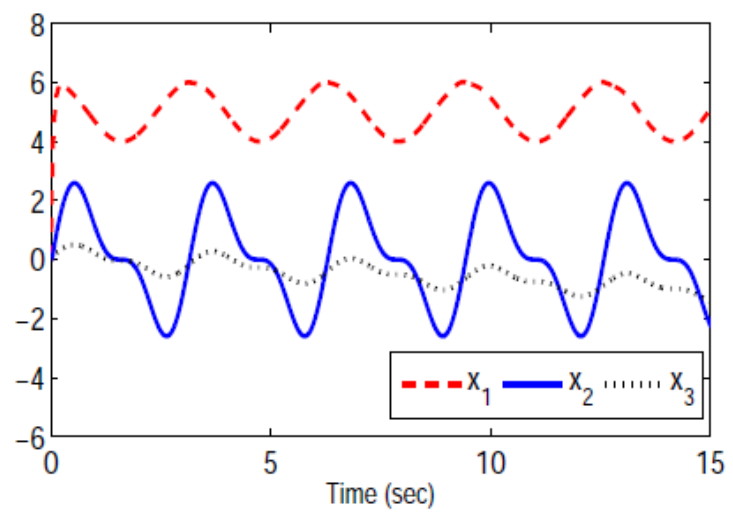

Figure 10. Internal state $\left(x_{1}, x_{2}, x_{3}\right)$

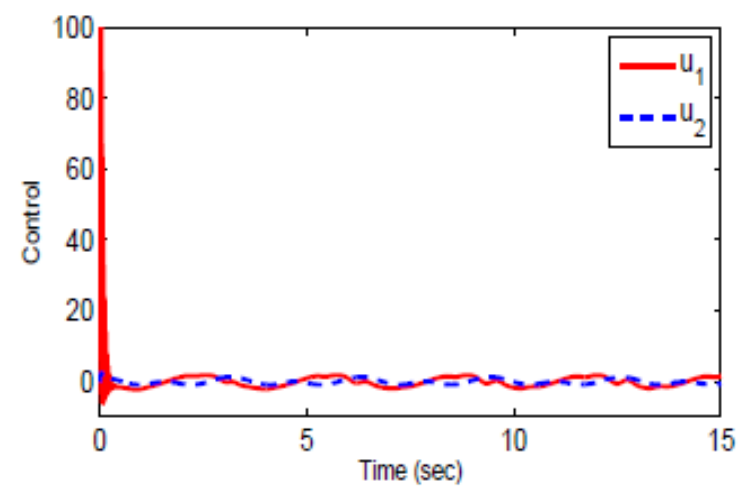

Figure 11. Input control signals $\left(u_{1}, u_{2}\right)$

\section{Conclusion}

This paper presents a new adaptive nonlinear optimal trajectory tracking recursive controller (ANORC) for non-affine in control nonlinear systems. The performance and robustness of this proposed ANORC have been validated and demonstrated by three different academic examples. This referred ANORC controller which employs only the output of the nonaffine in control nonlinear systems and a $L_{2}$ norm to minimize the trajectory tracking error has a simple architecture and can be implemented to the systems whose internal dynamics and parameters are unknown.

\section{Acknowledgment}

This work was partially supported by the National Natural Science Foundation of China (61304077, 61203115), by the Natural Science Foundation of Jiangsu Province (BK20130765), by the Specialized Research Fund for the Doctoral Program of Higher Education of China (20123219120038), by the Chinese Ministry of Education Project of Humanities and Social Sciences 
(13YJCZH171), by the Fundamental Research Funds for the Central Universities (30920130111014), and by the Zijin Intelligent Program of Nanjing University of Science and Technology (2013_ZJ_0105).

\section{REFERENCES}

1. Slotine, J. E., W. P. LI, Applied Nonlinear Control, Prentice Hall, Englewood Cliffs, New Jersey, 1991.

2. ISIDORI, A, Nonlinear Control Systems. 3rd edition, Springer-Verlag, London, 1995.

3. KHALIL, H. K., Nonlinear Systems. 3rd edition, Prentice Hall, Upper Saddle River, New Jersey, 2002.

4. DINH, H., S. BHASIN, D. KIM, W. E. DIXON, Dynamic Neural Network-based Global Output Feedback Tracking Control for Uncertain Second-order Nonlinear Systems, In American Control Conference, pages June 2012, pp. 6418-6423.

5. ZARGARZADEH, H., T. DIERKS, S. JAGANNATHAN, Optimal Adaptive Control of Nonlinear Continuous-time Systems in Strict Feedback Form with Unknown Internal Dynamics. In 51st IEEE Conference on Decision and Control, December 2012. Maui, Hawaii, USA, pp. 4127-4132.

6. PEREIRA DA SILVA, P. S., S. BATISTA, On State Representations of Nonlinear Implicit Systems. vol. 83, no. 3, 2010, pp. 441-456.

7. SONG, Y. D., Q. SONG, Survey of the Latest Developments in Control of Non-affine Systems. In Proceedings of the $30^{\text {th }}$ Chinese Control Conference, Yantai, China, 2011.

8. VRABIE, D., K. VAMVOUDAKIS, F. L. LEWIS, Adaptive Optimal Controllers based on Generalized Policy Iteration in a Continuous-time Framework, In Proceedings of the IEEE Mediterranean Conference on Control and Automation, 2009.

9. VRABIE, D., F. L. LEWIS, Neural Network Spproach to Continuous-time
Direct Adaptive Optimal Control for Partially Unknown Nonlinear Systems, Neural Networks, vol. 22, 2009.

10. TAO, J. E., P. KOKOTOVIC, Adaptive Control of Systems, John Wiley \& Sons, 1996.

11. LI, Z. H., M. KRSTIC, Optimal Design of Adaptive Tracking Controllers for Nonlinear Continuous Systems, vol. 33, no. 8,1997 , pp. 1459-1473.

12. WANG, H. P., B. KALCHEV, Y. TIAN, I. SIMEONOV, N. CHRISTOV, Modelling and Composed Recursive Model Free Control for the Anaerobic Digestion Process, In Advances in Intelligent Control Systems and Computer Science, Springer, January 2013, pp. 265-278.

13. KONCAR, V., C. VASSEUR, Control of Linear Systems using Piecewise Continuous Systems, vol. 150, no. 6, November 2003, pp. 565-576.

14. WANG, H. P., C. VASSEUR, V. KONCAR, A. CHAMROO, N. CHRISTOV, Sampled Tracking for Delayed Systems using Two Time-scales Sampled-data Controllers, Studies in Informatics and Control, vol. 19, no. 4, 2010, pp. 339-346.

15. WANG, H. P., C. VASSEUR, N. CHRISTOV, V. KONCAR, Vision Servoing of Robot Systems using Piecewise Continuous Controllers and Observers, Mechanical Systems and Signal Processing, vol. 33, December 2012, pp. 132-141.

16. LI, S. Z., H. P. WANG, Y. TIAN, N. CHRISTOV, A. AITOUCHE, NNPID based Stator Voltage Oriented Vector Control for DFIG, Studies in Informatics and Control, vol. 23, no. 1, 2014, pp. 5-12.

17. WANG, H. P., Y. TIAN, N. CHRISTOV. Piecewise Continuous Observers for Linear Systems with Sampled and Delayed Output. International Journal of System Science.

18. LIN, W., Time-varying Feedback Control of Nonaffine Nonlinear Systems without Drift, vol. 29, no. 2, 1996, pp. 101-110. 
